\title{
A New Look at Optimum Design for Convecting-Radiating Annular Fins of Trapezoidal Profile
}

\author{
B.T.F. Chung ${ }^{1, *}$, Y. Zhou ${ }^{2}$, Y. Wang ${ }^{3}$ and L.T. Yeh ${ }^{4}$ \\ ${ }^{1}$ University of Akron, Akron, $\mathrm{OH}, \mathrm{USA}$ \\ ${ }^{2}$ Bank of America, Los Angeles, CA, USA \\ ${ }^{3}$ Babcock \& Wilcox Inc. Barberton, OH, USA \\ ${ }^{4}$ Hua-Wei Technology Inc. Dallas, TX, USA
}

\begin{abstract}
This paper deals with a controversial problem in answering the question "Does the optimum fin design always exist? If not, what are the optimization ranges and limitations?" These authors employ a general example of convectingradiating trapezoidal annular fin with heat transfer at the tip and wall resistance at the interface. The present results indicate that the answer to the above first question is negative. The ranges of fin optimum design under different thermal and physical conditions are proposed. The effects of Biot number, radiation number, the heat loss at the tip, fin profile and overall wall resistance on fin optimization range are further investigated and discussed.
\end{abstract}

Keywords: Optimization range, trapezoidal fin, convection and radiation, wall resistance, tip heat loss.

\section{INTRODUCTION}

The utilization of fins is an effective method to enhance the heat dissipation from a surface. Applications for finned surfaces are widely seen in air-conditioning and refrigeration, aerospace, chemical processing plants, and in the thermal control of electronic and electrical devices. There are various types of fins available in industry. Among them, annular fins are especially important for compact heat exchangers; fin with trapezoidal profile has the most practical shape. From the thermal designer's point of view, it is of significance to search for an optimum fin design.

There are two categories of optimization that pertain to single fin design. The first category of optimization is to determine the best profile and dimensions that yield minimum weight or mass for a specified heat flow and a given fin shape (e.g. longitudinal, radial and pin fins). A solution was first proposed by Schmidt [1] authenticated by Duffin [2], which was further extended by Maday [3] and Hanin and Campo [4]. However, the mathematical solutions to these kinds of optimum design resulted in fin profiles with sharp curved surfaces which are difficult or costly to fabricate. Therefore one alternative way is to fix a suitable simple profile (e.g. rectangular, triangular, parabolic, trapezoidal, etc) and then determine the dimensions of the fin so that it dissipates the maximum amount of heat for a given amount of mass. The present work falls into the second category.

*Address correspondence to this author at the Department of Mechanical Engineering, The University of Akron, Akron, Ohio, 44325-3903, USA; Tel: 330-972-7739; E-mail: bchung @uakron.edu
The studies of the optimum dimensions for purely convective fins have yielded numerous publications in which various fin shapes were employed. Among them only Chung et al. [5] and Razelos and Imre [6] considered the trapezoidal profile. Since radiation heat transfer and free convection play equally important role in most of the practical applications except for some special cases (e.g. outer space), the neglect of radiation may cause significant errors in the calculations of optimum fin dimensions. In so far as convecting-radiating fins are concerned, few optimum studies are available in the literature. Zubair and Khan [7] employed existing software to obtain the optimal dimensions of convecting-radiating annular fins with curved surfaces. However fins with curved surfaces are difficult and expensive to fabricate.

Fins with a constant slope profile, or trapezoidal profile, are widely used in engineering applications because they can be easily fabricated. As pointed out in a review paper by Aziz [8], the optimum convecting-radiating annular fins call for more research endeavors. More recently, Chung and Zhou [9] studied the optimum designs for the convectingradiating annular fins of trapezoidal profile with heat transfer at the fin tip and wall thermal resistance at the fin base. These authors presented a parametric study, fin effectiveness and optimum design charts but without providing any information on the optimization ranges of the annular fin. Here the term "optimization range" indicates the ranges of geometric and thermal parameters of the annular fin for which optimum fin design is available.

A critical question which has not been responded by the previous investigators is "Will the optimum design always 
exist?" By assuming the negligible heat loss at the fin tip and the negligible curvature effects, most of previous studies implied that the fin optimum designs are always obtainable. Meanwhile, the general reviews by Aziz and Kraus [10] and Razelos [11] as well as a current treatise of extended surface heat transfer by Kraus et al. [12] have not pointed out the otherwise. Laor and Kalman [13] specifically claimed that the optimum design always exists. Although the authors also considered the tip heat loss in the study of rectangular annular fins, they drew the above conclusion by referring to their previous work [14], which neglected heat loss at the fin tip. Therefore, the claim and the corresponding optimum results with convective fin tips shown in Fig. (9) of Laor and Kalman [13] appear to be questionable. A series of studies on optimal straight fins and spines by Chung and Iyer [15] and Yeh [16] imply that the optimum designs of extended surfaces are only available in certain ranges of characteristic variables. However, none of these investigations pinpointed the domain and limitations of the optimum designs.

The purpose of this work is to further extend the previous research by Chung and Zhou [9] by responding to the question posted in the beginning of the previous paragraph. It is hoped that the optimization ranges proposed in the present study will be helpful in analyzing and designing annular fins subjected to convection and radiation at the boundary.

\section{ANALYSIS}

Consideration is given to the optimization of a single annular trapezoidal fin dissipating heat by simultaneous convection and radiation. In the present analysis, the conventional assumption of specifying the fin base wall temperature (the boundary condition of the first kind) is abandoned, since in practice, only the fluid temperature inside the annular fin tube and the surrounding temperature outside the fin are known. Instead of insulation at the fin tip as postulated from Murray-Gardner assumptions, tip heat transfer by convection and radiation is included in this study. Furthermore, the commonly used length of arc idealization is discarded i.e., the length of arc effect of fin profile is taken into account in the present investigation. The following additional commonly used assumptions are applied to the present analysis:

1) The heat conduction in the fin is one-dimensional and steady state. 2) The fin material is homogeneous and isotropic. 3) There is no heat generation inside the fin. 4) The heat transfer coefficient is constant over the surface of the fin. 5) The ambient fluid temperature is uniform, and so is the fluid temperature inside the pipe to which the fin is appended. 6) Fin to fin and fin to base radiation interaction are neglected.

For the convenience of the reader and to maintain the continuity of this presentation, a brief analysis will be provided below although the same governing equations can be found in Chung and Zhou [9].

\subsection{Governing Equation and Boundary Conditions}

Considering an annular trapezoidal fin shown in Fig. (1), the fin profile function is expressed by the following form

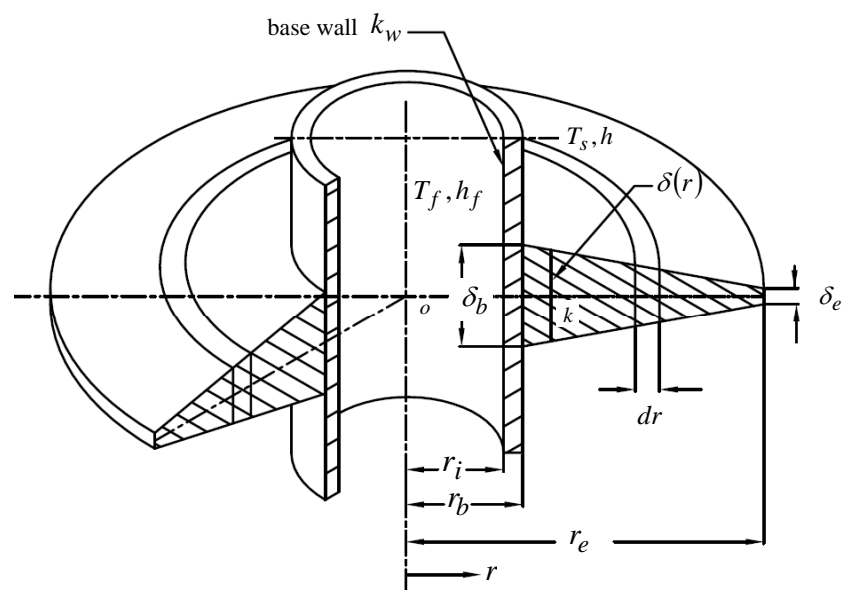

Fig. (1). Schematic of an Annular Fin with Trapezoidal Profile.

$$
\delta(r)=\delta_{e}+2\left(r_{e}-r\right) \tan \varphi
$$

Where $\phi$ is the taper angle of the trapezoidal fin.

Considering the constant properties of fin material, the heat balance for a control volume of a finite length, dr, results in

$\frac{d}{d r}\left[r \delta(r) \frac{d T}{d r}\right]=\frac{2}{\cos \varphi}\left[\frac{h r}{k}\left(T-T_{a}\right)+\frac{\sigma \varepsilon r}{k}\left(T^{4}-T_{s}^{4}\right)\right]$

Due to steady state heat exchange, the total heat flux from the fluid inside the primary pipe to the fin base is constant. Considering convection at the inner surface of pipe, conduction within the wall of pipe, and contact thermal resistance at the fin base, the boundary condition at fin base is modeled as

$$
-k \frac{d T}{d r}=\frac{T_{f}-T}{\frac{r_{b}}{h_{f} r_{i}}+\frac{r_{b} \ln \left(r_{b} / r_{i}\right)}{k_{w}}+R_{t c}} \quad @ \quad \text { } r=r_{b}
$$

Where $R_{t c}$ is the thermal contact resistance; $h_{f}$ is the heat transfer coefficient inside the pipe and $r_{b}$ is the fin base radius. The boundary condition of this type was first introduced by Aziz [17] for straight fins and further developed by Chung et al. [5].

Because the tip heat transfer area is not zero, both convection and radiation from the tip of trapezoidal fins are taken into account. Therefore, the boundary condition at the fin tip is

$-k \frac{d T}{d r}=h_{e}\left(T-T_{a}\right)+\sigma \varepsilon\left(T^{4}-T_{s}^{4}\right) \quad @ r=r_{e}$ 
Where, in general, the heat transfer coefficient at the fin tip, $h_{e}$, may not be the same as the average heat transfer coefficient over the fin surface, h. Without the loss of generality, the ambient temperature $T_{a}$ is set equal to environment temperature $T_{s}$ in this analysis. For the case of zero heat transfer area at the tip (triangular fin), Eq. (4) is replaced by $q=0 @ r=r_{e}$

Introducing the following normalized variables and parameters into Equations (1) - (4)

$$
\begin{gathered}
\rho=\frac{r_{e}}{r_{b}}, \quad \lambda=\frac{\delta_{e}}{\delta_{b}}, \quad \xi=\frac{r}{r_{b}}, \\
\theta=\frac{T}{T_{f}}, \quad \theta_{s}=\frac{T_{s}}{T_{f}}, \quad \theta_{b}=\frac{T_{b}}{T_{f}}, \\
A=\frac{\rho-\lambda}{\rho-1}, \quad B=\frac{\lambda-1}{\rho-1}
\end{gathered}
$$

and substituting Equation (1) into Equation (2) results in the following non-dimensional governing equation:

$$
\begin{aligned}
& (A+B \xi) \frac{d^{2} \theta}{d \xi^{2}}+\left(\frac{A}{\xi}+2 B\right) \frac{d \theta}{d \xi}=\frac{2}{\cos \varphi} \frac{r_{b}}{\delta_{b}} \\
& {\left[\frac{h r_{b}}{k}\left(\theta-\theta_{s}\right)+\frac{\sigma \varepsilon T_{f}^{3} r_{b}}{k}\left(\theta^{4}-\theta_{s}^{4}\right)\right]}
\end{aligned}
$$

Where $\rho$ is radius ratio, $\lambda$ is taper ratio, $\theta_{s}$ and $\theta_{b}$ represent normalized ambient and fin base temperatures respectively. The corresponding non-dimensional boundary conditions become

$$
\begin{array}{ll}
\left.\frac{d \theta}{d \xi}\right|_{\xi=1}=\frac{\theta-1}{\frac{k}{h_{f} r_{i}}+\frac{k \ln \left(r_{b} / r_{i}\right)}{k_{w}}+\frac{k R_{t c}}{r_{b}}} \quad @ \xi=1 & \\
\left.\frac{d \theta}{d \xi}\right|_{\xi=\rho}=\frac{h_{e} r_{b}}{k}\left(\theta_{s}-\theta\right)+\frac{\sigma \varepsilon T_{f}^{3} r_{b}}{k}\left(\theta_{s}^{4}-\theta^{4}\right) & @ \xi=\rho
\end{array}
$$

It is advantageous to introduce the taper ratio, $\lambda$, because, geometrically, a fin with rectangular profile (constant thickness) can be represented when $\lambda$ is set equal to 1 ; triangular profile (sharp end) can be described when $\lambda$ is 0 , and different trapezoidal profiles can be approached when $\lambda$ is somewhere between 0 and 1 . The two dimensionless geometry parameters, $\mathrm{A}$ and $\mathrm{B}$, are introduced to simplify the expression of the governing equation.

Another important geometrical parameter in the governing equation is the taper angle, $\phi$. In almost all of the work cited in the literature, the "length of arc" idealization was employed. The term $\cos \phi$ in the governing equation represents the length of arc effect. From a simple geometry, we can write:

$$
\frac{1}{\cos \varphi}=\sqrt{1+\left(\frac{B}{2} \frac{\delta_{b}}{r_{b}}\right)^{2}}
$$

The fin volume, as shown in Fig. (1), is given by

$$
\begin{array}{r}
\mathrm{v}=\int_{r_{b}}^{r_{e}} 2 \pi r \delta(r) d r=\int_{1}^{\rho} 2 \pi r_{b} \xi \delta_{b}(A+B \xi) d\left(r_{b} \xi\right) \\
=2 \pi r_{b}^{2} \delta_{b}\left[\frac{A}{2}\left(\rho^{2}-1\right)+\frac{B}{3}\left(\rho^{3}-1\right)\right]
\end{array}
$$

Two dimensionless geometry parameters are defined by Equations (9a) and (9b):

$$
G=2\left[\frac{A}{2}\left(\rho^{2}-1\right)+\frac{B}{3}\left(\rho^{3}-1\right)\right], \quad V=\frac{\mathrm{v}}{\pi r_{b}^{3}}
$$

Introducing $\mathrm{G}$ and $\mathrm{V}$ into Equation (9) results in

$$
\frac{\delta_{b}}{r_{b}}=\frac{V}{G}
$$

Substituting Equations (8)-(10) into Equation (5) yields

$$
\begin{aligned}
& (A+B \xi) \frac{d^{2} \theta}{d \xi^{2}}+\left(\frac{A}{\xi}+2 B\right) \frac{d \theta}{d \xi} \\
& =\sqrt{B^{2}+\left(\frac{2 G}{V}\right)^{2}}\left[m_{c}\left(\theta-\theta_{s}\right)+m_{r}\left(\theta^{4}-\theta_{s}^{4}\right)\right]
\end{aligned}
$$

Where $m_{c}$ is the convective characteristic number (or Biot number) and $m_{r}$ is the radiation characteristic number. They are defined as

$$
\begin{aligned}
& m_{c}=\frac{h r_{b}}{k} \\
& m_{r}=\frac{\sigma \varepsilon T_{f}^{3} r_{b}}{k}
\end{aligned}
$$
tip is

Similarly, the convective characteristic number at the fin $m_{c, e}=\frac{h_{e} r_{b}}{k}$

and also

$$
\beta=\frac{m_{c, e}}{m_{c}}=\frac{h_{e}}{h}
$$

Note that $\beta$ was set equal to unity in the most previous analyses. Since the free convection and thermal radiation are both direction and geometric dependent, $\beta$ can be different from unity. The overall wall resistance at the fin base is written as

$R_{w}=\frac{k}{h_{f} r_{i}}+\frac{k \ln \left(r_{b} / r_{i}\right)}{k_{w}}+\frac{k R_{t c}}{r_{b}}$ 
Introducing the parameters $m_{r}, m_{c}$ and $R_{w}$ into Equations (6) and (7), yields respectively the non-dimensional boundary conditions of the forms:

$\frac{d \theta}{d \xi}=\frac{\theta-1}{R_{w}} \quad @ \xi=1$

$\frac{d \theta}{d \xi}=m_{c, e}\left(\theta_{s}-\theta\right)+m_{r}\left(\theta_{s}^{4}-\theta^{4}\right) @ \xi=\rho$

The system is specified by the nonlinear differential equation, Equation (11), subjected to the nonlinear boundary conditions, represented by Equations (17)-(18).

In the present optimization process, the optimal geometry of fin will be pursued under specified thermal conditions. As shown in Equations (9)-(10) and the definitions of $\mathrm{A}$ and $\mathrm{B}$, the annular trapezoidal fins with a given volume can be completely described by $\rho, \lambda$ and $\delta_{b} / r_{b}$. Therefore, the optimization design is achieved, once the optimal values of $\rho^{*}$ and $\delta_{b} * / r_{b}$ are found.

\subsection{Heat Dissipation}

In the steady state, the heat dissipation from the fin surface is equal to the heat transfer at the fin base. It can be written as

$q=-\left.k A_{b} \frac{d T}{d r}\right|_{r=r_{b}}=-\left.k\left(2 \pi \delta_{b}\right) T_{f} \frac{d \theta}{d \xi}\right|_{\xi=1}$

This can be rearranged into the following nondimensional form:

$Q=\frac{q}{k\left(2 \pi r_{b}\right) T_{f}}=-\left.\frac{\delta_{b}}{r_{b}} \frac{d \theta}{d \xi}\right|_{\xi=1}=-\left.\left(\frac{V}{G}\right) \frac{d \theta}{d \xi}\right|_{\xi=1}$

\subsection{Optimum Design}

From their definitions, the geometry parameters A, B and $\mathrm{G}$ only involve $\rho$ and $\lambda$. In most cases, $\lambda$ is specified by the designer. (The rationale was presented below Eq. (7) in section 2.1) Thus, for an annular trapezoidal fin with the given volume, the governing equation involves the geometry parameters A, B and G, which are solely expressed in terms of a single variable, $\rho$. Generally, the maximum dimensionless heat flux will be found by solving equation for $\mathrm{Q}$, or $(d \theta / d \xi)_{\xi=1}$, and then, setting $d Q / d \rho=0$. However, since Equation (11) is a non-linear second order differential equation, analytical solutions for $\mathrm{Q}$ and $d Q / d \rho=0$ are highly unfeasible.

To evaluate temperature profile and heat dissipation numerically, the fourth order Runge-Kutta method along with the shooting method have been employed. It is obvious that, fins may have different geometry profiles (say $\rho$ ), even though they have same volume. The Golden Section Search method is used to pinpoint the maximum heat flux and the corresponding optimal $\rho^{*}$ for a given volume. In other words, optimization of the fin with a given volume is done by searching the maximum heat dissipation at the corresponding $\rho^{*}$ and then, substituting $\rho^{*}$ into Equation (10) to obtain the optimal non-dimensional fin base thickness, $\delta_{b} * / r_{b}$.For further details regarding Golden Section Search method, the reader may refer to the text by Daniel [18].

\section{RESULTS AND DISCUSSION}

Although the above analyses are for the case of fixed fin volume, they can be equally applied to the case of specified heat dissipation with an additional iterative scheme. As mentioned earlier, this paper focuses to the ranges of optimization of convecting-radiating annular fins of trapezoidal profile.

\subsection{Temperature Distribution}

Temperature distributions along the fin with various fin lengths are demonstrated in Figs. (2 and 3) for two different cases. The normalized position along the trapezoidal fin is given as

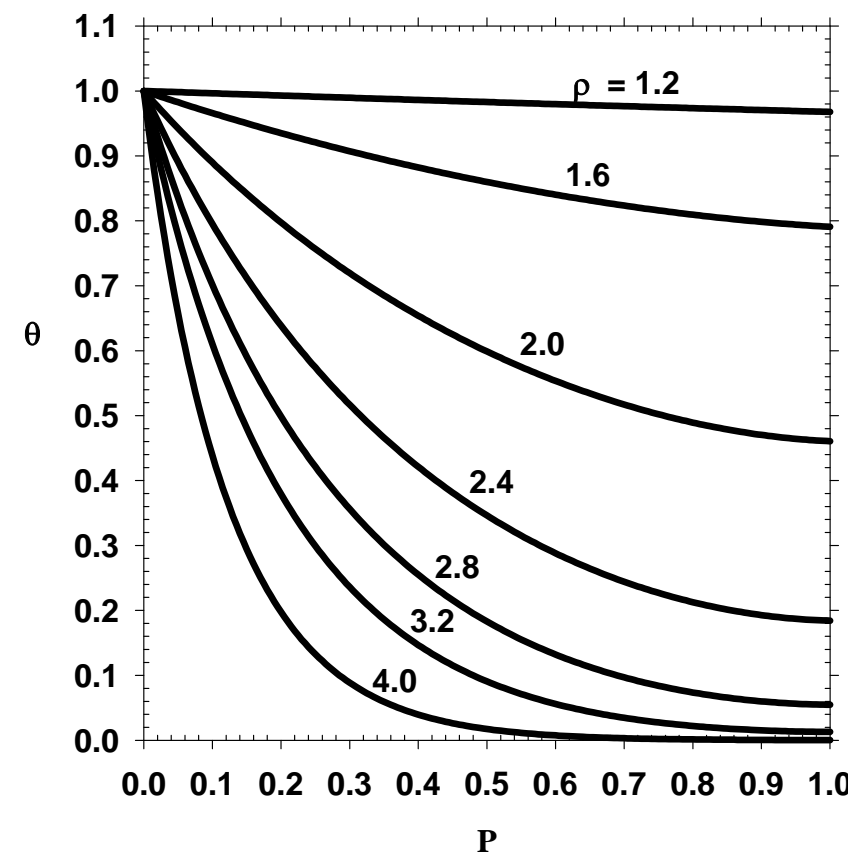

Fig. (2). The Dimensionless Temperature Distributions along the Fin without Wall Resistances $\left(\mathrm{m}_{\mathrm{r}}=0.05, \mathrm{~m}_{\mathrm{c}}=0.1, \beta=1, \mathrm{R}_{\mathrm{w}}=0, \theta_{\mathrm{s}}\right.$ $=0, \lambda=0.5, \mathrm{~V}=0.3$ ).

$\mathrm{P}=(\xi-1) /(\rho-1)$, where $\mathrm{P}$ equal 0 and 1 represent the nodes at the fin base and the tip respectively.

For the case shown in Fig. (2), the overall base wall resistance is neglected and the environment temperature is at the absolute zero; the fin is subjected to simultaneous 
convection and radiation. A similar case is given in Fig. (3) except that the environment temperature is non-zero and the wall resistance is not neglected. In both cases, the temperature gradient at the fin base increases considerably as the fin length increases. However the dimensionless temperature decreases from unity at the fin base to a certain value at the fin tip when the wall resistance is neglected but the base temperature is always less than 1 in Fig. (3) due to the effect of the wall resistance.; when the fin length increases, the temperature at the fin base decreases but its gradient at wall increases.

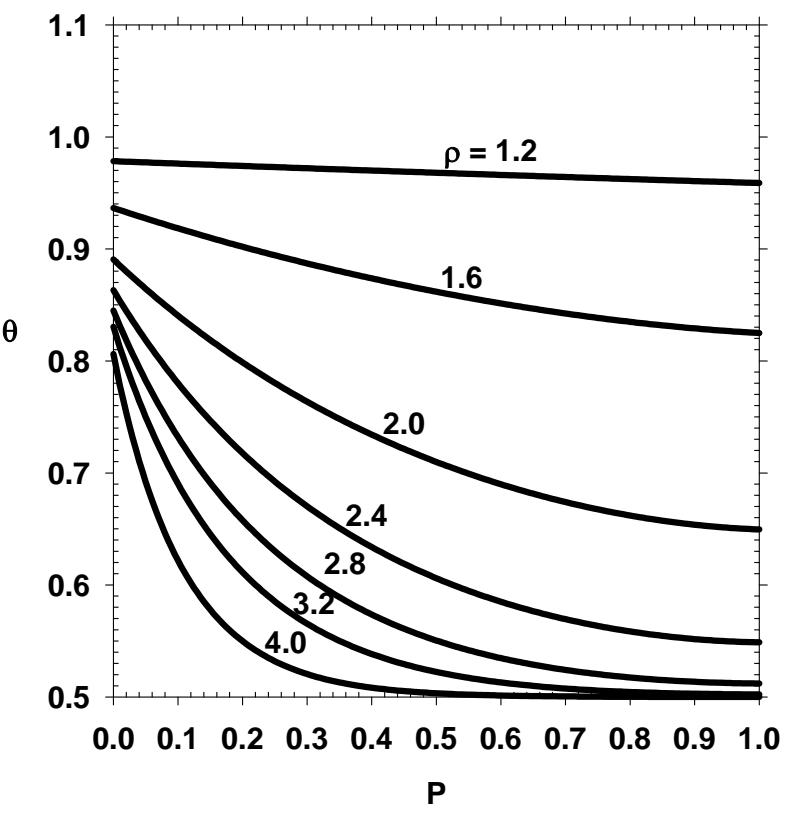

Fig. (3). The Dimensionless Temperature Distributions along the Fin with Wall Resistances $\left(\mathrm{m}_{\mathrm{r}}=0.05, \mathrm{~m}_{\mathrm{c}}=0.1, \beta=1, \mathrm{R}_{\mathrm{w}}=0.2, \theta_{\mathrm{s}}=\right.$ $0.5, \lambda=0.5, \mathrm{~V}=0.3)$.

\subsection{Heat Dissipation}

The dimensionless heat transfer rate, $Q$ as a function of radius ratio, $\rho$, is illustrated in Fig. (4). It is observed that $\mathrm{Q}$ becomes unbounded when $\rho$ is near 1 (the fin length is 0 when $\rho$ is 1 ). The reason for this phenomenon can be explained by observing the definition of non-dimensional heat transfer rate, $Q=-\left(\delta_{b} / r_{b}\right)(d \theta / d \xi)_{\xi=1}$. Because the fin volume, $\mathrm{v}$, is constant in the present work, $\delta_{b}$ which represents the heat transfer area at the fin base, will increase while $\rho$ decreases. On the other hand, the temperature gradient at the fin base, $-(d \theta / d \xi)_{\xi=1}$, will decrease while $\rho$ decreases. Therefore, $Q$ is the product of these two components that always have opposite trends. It is shown from our numerical calculations, as $\rho$ approaches 1 , the increase of $\delta_{b}$ is more rapid than the decrease of $-(d \theta / d \xi)_{\xi=1}$. In other words, an extremely large $Q$ is caused by $\delta_{b}$, or the heat transfer area at fin base. Obviously, the designs in this case are impractical, though $Q$ seems to be extremely large.

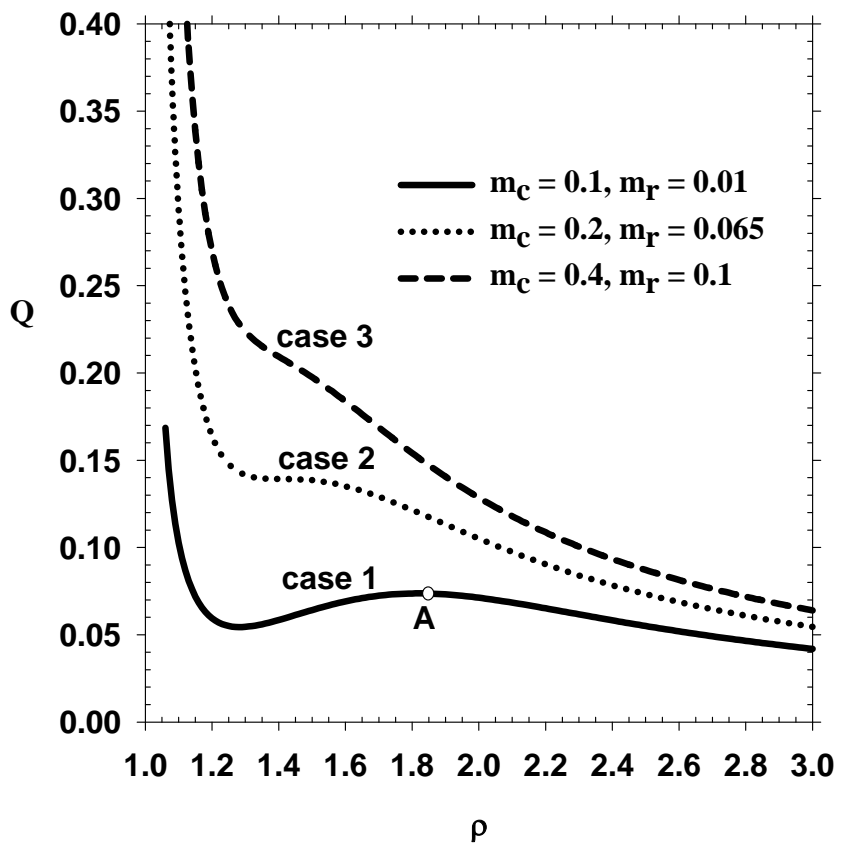

Fig. (4). The Heat Dissipation Rate, Q, as a Function of the Annular Fin Radius Ratio, $\rho\left(\beta=1, R_{w}=0.2, \theta_{s}=0.5, \lambda=0.5, V=0.3\right)$.

\subsection{Numerical Comparison with a Limiting Case}

The accuracy of the present numerical solutions will be examined by comparing an existing limiting case. Kern and Kraus [19] presented solutions for the heat transfer rate from a radial fin with an insulated tip and without wall resistance; the fin radiates heat at a non-zero environment temperature. Three different fin profiles were considered by the authors, namely, rectangular, trapezoidal and triangular profiles. Table 1 shows the comparison of the predicted heat transfer rate between the present study and the Example 4.9 "Radiation from radial fins" in Kern and Kraus [19]. The excellent agreement is found and the minor numerical differences which are probably because the different computer software and hardware were employed in both studies.

Table 1. Comparison of Heat Dissipation (W) between the Present Limiting Solutions and Those of Kern and Kraus [19]

$\left(\rho=2.5, \delta_{b} / r_{b}=1 / 16, m_{c}=0, m_{r}=0.0025, \theta_{s}=0.2^{1 / 4}, R_{w}=0\right.$, insulated fin tip)

\begin{tabular}{|c|c|c|c|}
\hline & $\begin{array}{c}\text { Rectangular } \\
(\boldsymbol{\lambda}=\mathbf{1})\end{array}$ & $\begin{array}{c}\text { Trapezoidal } \\
(\boldsymbol{\lambda}=\mathbf{0 . 5})\end{array}$ & $\begin{array}{c}\text { Triangular } \\
(\boldsymbol{\lambda}=\mathbf{0})\end{array}$ \\
\hline \hline Present work & 54.13 & 53.34 & 51.40 \\
Kern \& Kraus & 54.42 & 53.34 & 51.34 \\
\hline
\end{tabular}




\subsection{Optimization Ranges}

Another important phenomenon shown in Fig. (4) is that the maximal heat transfer rate, $\mathrm{Q}^{*}$, is not always obtainable. In case $1\left(m_{c}=0.1, m_{r}=0.01\right), Q$ at point $\mathrm{A}(\rho=1.82)$ is the maximum, because $(d Q / d \rho)=0$ there. However, in case $3\left(m_{c}=0.4, m_{r}=0.1\right)$, there is apparently no maximum by definition. Case $2\left(m_{c}=0.2, m_{r}=0.065\right)$ can be treated as a boundary case between the cases with maximum and without maximum. It is obvious that $m_{c}$ and $m_{r}$ have strong influences on this boundary. When $m_{c}$ and $m_{r}$ are small, the maximum is available. However, when $m_{c}$ and $m_{r}$ are beyond certain values, the maximum is not obtainable. These ranges are referred to as the optimization ranges in the present work.

As mentioned earlier, three more realistic considerations in the present study, which were usually neglected in many previous studies, are the inclusions of (1) the heat loss at fin tips, (2) the fin curvature effects, and (3) the wall thermal resistances. Further investigations in the present study indicate that, if both heat loss at tips and curvature effects are neglected, the optimum heat flux is always available. As illustrated in Fig. (5), the heat transfer increases from 0 at $\rho=1$ and then decreases, if both heat loss at the tip and the curvature effects are neglected; this implies that there always exists an optimal Q*. However, if either the heat loss at the fin tip or the curvature effect is applied, the heat transfer will decrease from infinity at $\rho=1$ instead of increasing from 0 . For the reason mentioned above (see Fig. 4), the optimal designs are not obtainable under some circumstances. For the rectangular fin $(\lambda=1)$, which has no curvature effects, the phenomenon of lack of optimum designs occurs due to the inclusion of heat loss at the fin tip.

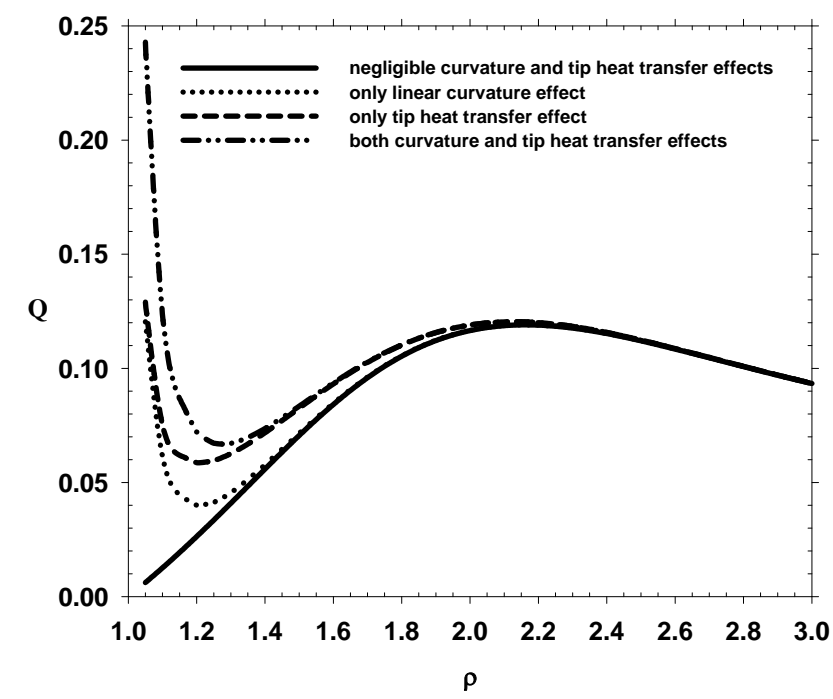

Fig. (5). Curvature and Tip Heat Transfer Effects on Heat Dissipation $\left(\mathrm{m}_{\mathrm{r}}=0.01, \mathrm{~m}_{\mathrm{c}}=0.05, \theta_{\mathrm{s}}=0, \mathrm{R}_{\mathrm{w}}=0, \mathrm{~V}=0.3\right)$.
On the other hand, for the triangular fin $(\lambda=0)$, which has no heat loss at the fin tip; the same phenomenon still occurs due to the inclusion of curvature effects. This implies that either of curvature effects or heat loss at the fin tip will create an optimization range, or a limitation of optimum fin.

By examining Fig. (4), it is found that non-optimum zones occurs when $m_{c}$ and $m_{r}$ are larger than certain values, which are referred to as the optimization range of $m_{c}$ and $m_{r}$ in the present study. In this paper, the optimization ranges of $m_{c}$ and $m_{r}$ are presented and shown at different parameters of $R_{w}, \beta, \theta_{s}, \mathrm{~V}$ and $\lambda$. Furthermore, the effects of these parameters on the optimization range are examined. As will be seen later, the optimization range is the area bounded by a parameter curve and two abscissas which correspond to Biot number and radiation number in the present case.

\subsubsection{Effect of Overall Thermal Resistance}

Figs. (6 to 8) show the effect of $R_{w}$ on this range. It is found that, for all of three different shapes (triangular, a typical trapezoidal and rectangular), the ranges of $m_{c}$ and $m_{r}$ shrink with the increase of $R_{w}$. For example, as shown in Fig. (7), under the pure radiation $\left(m_{c}=0\right)$, the optimum design is not available if $m_{r}$ is beyond 0.156 when $R_{w}=0.1$. But at $R_{w}=0.8$ the optimum design is not obtainable even when $m_{r}$ is greater than 0.056 . The trend is the same for the pure convection $\left(m_{r}=0\right)$. For the combined mode of radiation and convection, it is shown in the same figure that at $R_{w}=0.05$ and $m_{r}=0.095$, the optimal dimensions are

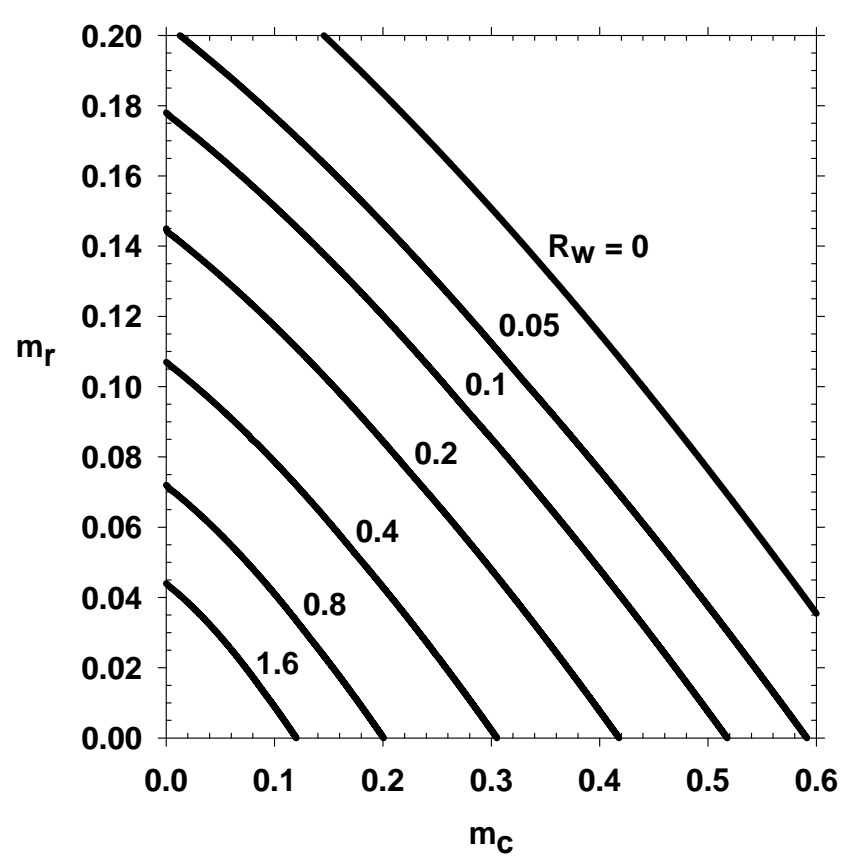

Fig. (6). The Optimization Ranges of $m_{c}$ and $m_{r}$ as a Function of $R_{w}$ for a Triangular Annular Fin $\left(\beta=1, \theta_{\mathrm{s}}=0.5, \mathrm{~V}=0.3\right)$. 


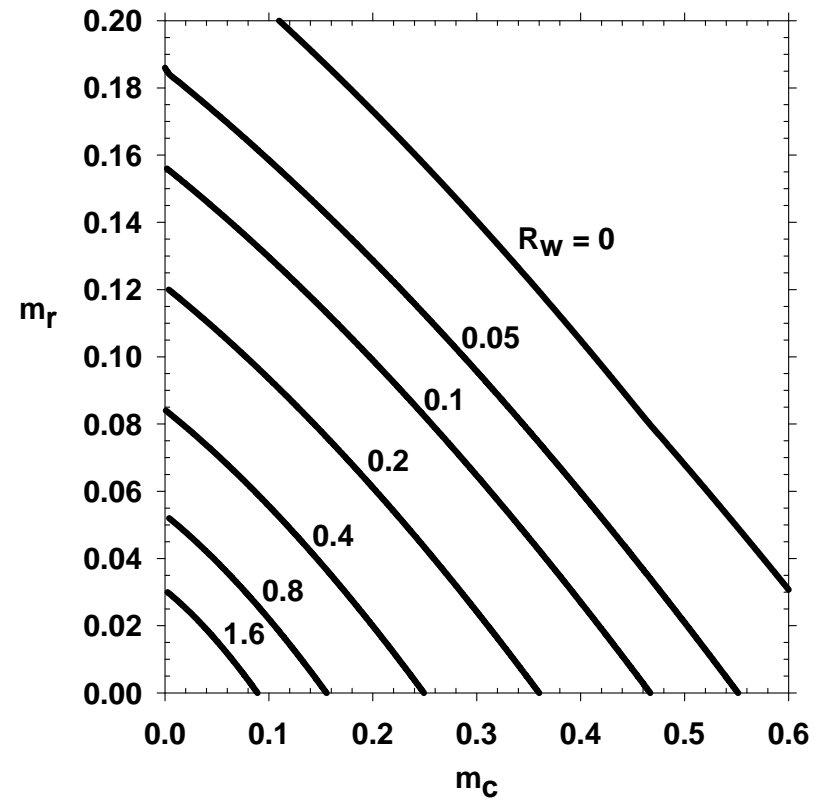

Fig. (7). The Optimization Ranges of $m_{c}$ and $m_{r}$ as a Function of $R_{w}$ for a Typical Trapezoidal Annular Fin $\lambda=0.5\left(\beta=1, \theta_{\mathrm{s}}=0.5, \mathrm{~V}=0.3\right)$.

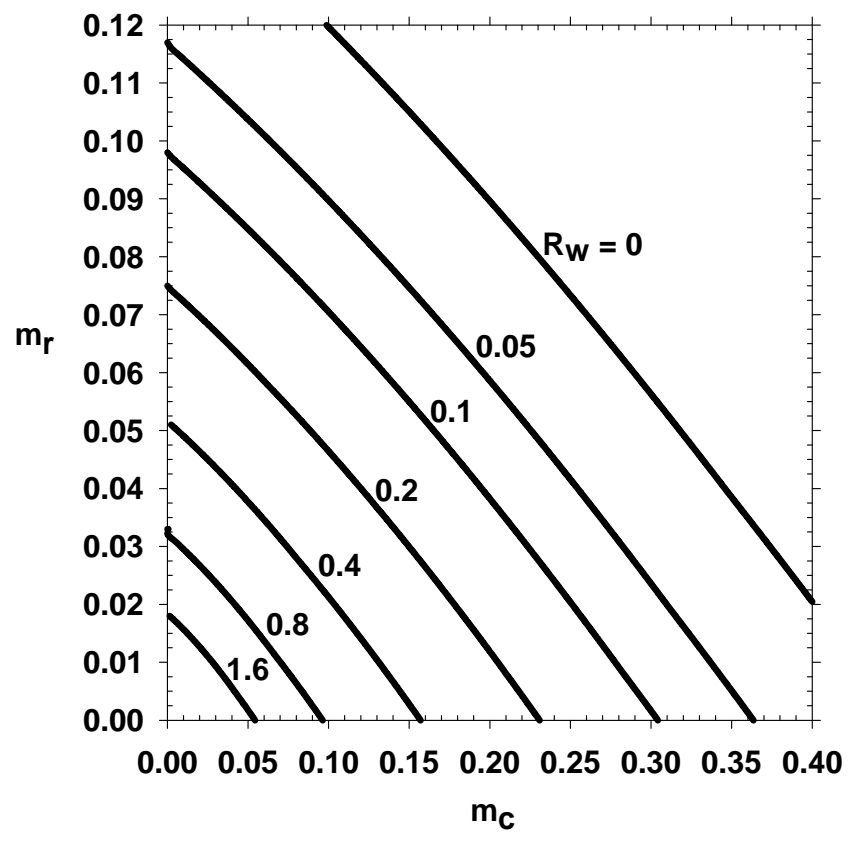

Fig. (8). The Optimization Ranges of $m_{c}$ and $m_{r}$ as a Function of $R_{w}$ for a Rectangular Annular Fin $\left(\beta=1, \theta_{\mathrm{s}}=0.5, \mathrm{~V}=0.3\right)$.

available only if $m_{c}$ is less than 0.3 . Also comparison among Figs. (6 to 8 ), as $\lambda$ increases from 0 to 1 for a specific $R_{w}$, both optimization ranges of $m_{r}$ and $m_{c}$ decrease.

\subsubsection{Effect of Tip Heat Transfer}

The optimization ranges of $m_{c}$ and $m_{r}$ for a trapezoidal and the rectangular fins are plotted for various values of $\beta$ in
Figs. (9 and 10). Obviously, there is no variation of $\beta$ for triangular fins $(\lambda=0)$, because there is no heat loss at the fin tip. In both figures, all curves are coincident at $m_{c}=0$, since $\beta$ is independent of pure radiation conditions. It is observed that the optimization ranges of $m_{c}$ and $m_{r}$ decrease while $\beta$ increases. In other words, the less the heat loss at the fin tip, the bigger the optimization ranges of $m_{c}$ and $m_{r}$.

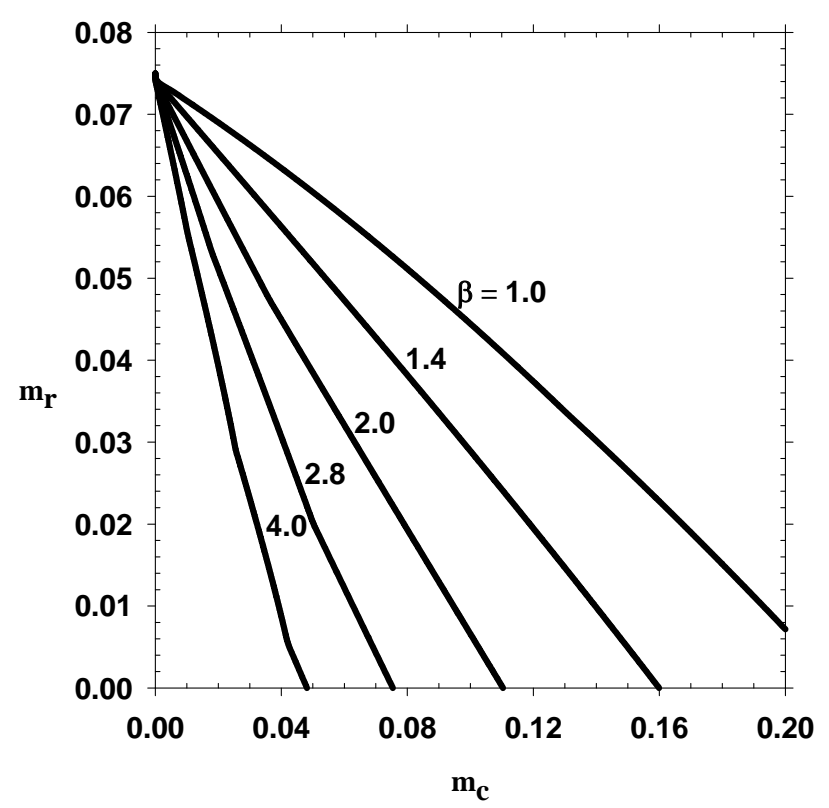

Fig. (9). The Optimization Ranges of $m_{c}$ and $m_{r}$ as a Function of $\beta$ for a Typical Trapezoidal Annular Fin of $\lambda=0.5\left(\mathrm{R}_{\mathrm{w}}=0.5\right.$, $\theta_{\mathrm{s}}=0.5, \mathrm{~V}=0.3$ ).

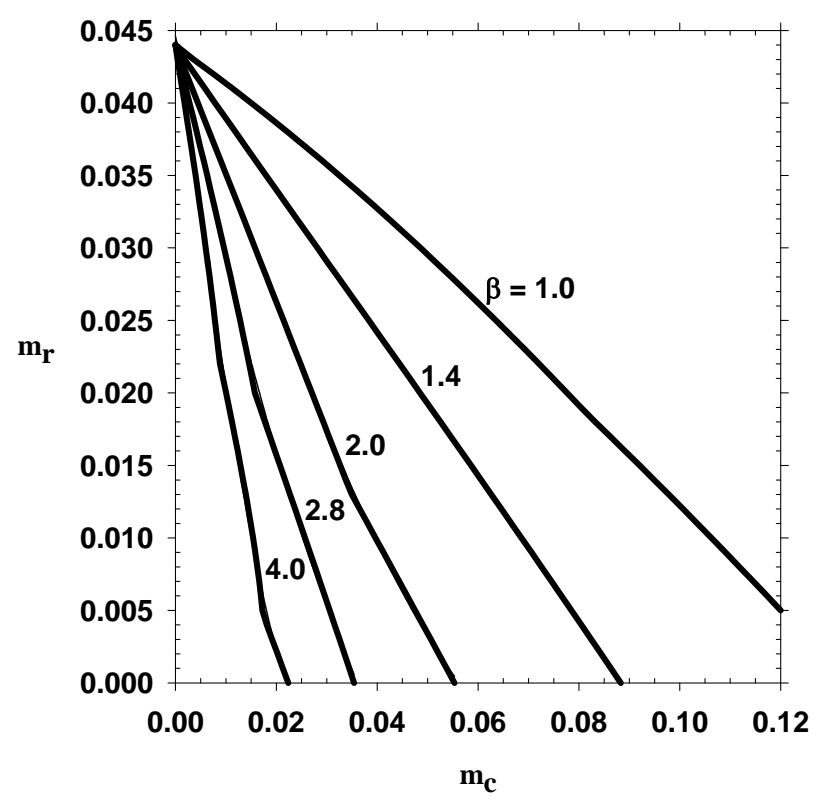

Fig. (10). The Optimization Ranges of $m_{c}$ and $m_{r}$ as a Function of $\beta$ for a Rectangular Annular Fin $\left(\mathrm{R}_{\mathrm{w}}=0.5, \theta_{\mathrm{s}}=0.5, \mathrm{~V}=0.3\right)$. 


\subsubsection{Effects of Ambient Temperature}

The effects of the ambient temperature $\theta_{s}$ on the optimization ranges are depicted in Figs. (11 to 13). It is shown that the higher the temperature difference between inner pipe fluid and the ambient, the larger the optimization ranges of $m_{c}$ and $m_{r}$. When the temperature difference is not too high (e.g. $\theta_{s}>0.8$ ), the curves are linear. All curves end at the same point at $m_{r}=0$, because the effects of $\theta_{s}$ disappear under the pure convection condition at which the problem becomes linear (i.e., $\theta-\theta_{s}$ can be replaced by a

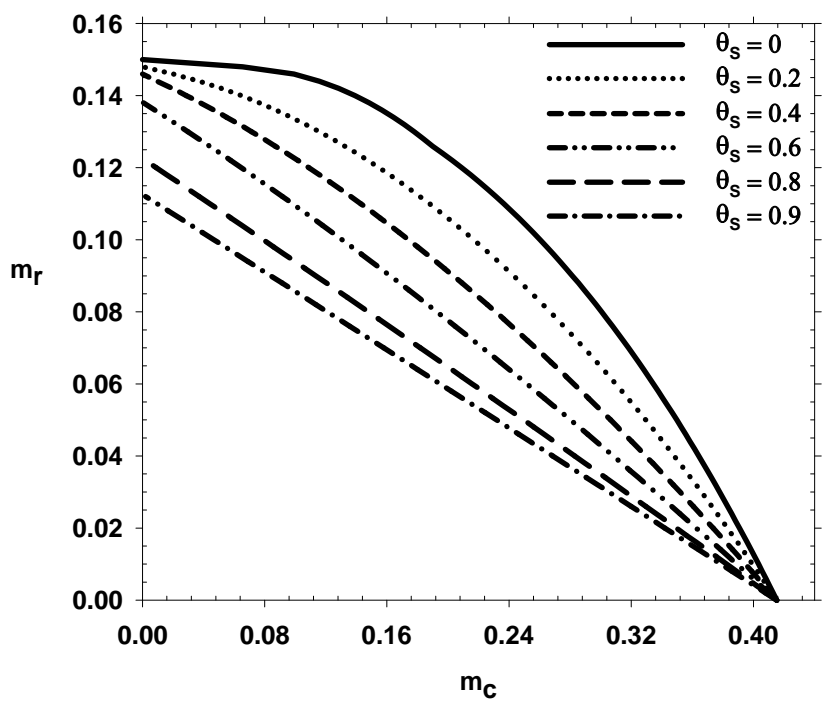

Fig. (11). The Optimization Ranges of $\mathrm{mc}$ and $\mathrm{mr}$ as a Function of $\theta_{\mathrm{s}}$ for a Triangular Annular Fin $\left(\beta=1, \mathrm{R}_{\mathrm{w}}=0.2, \mathrm{~V}=0.3\right)$.

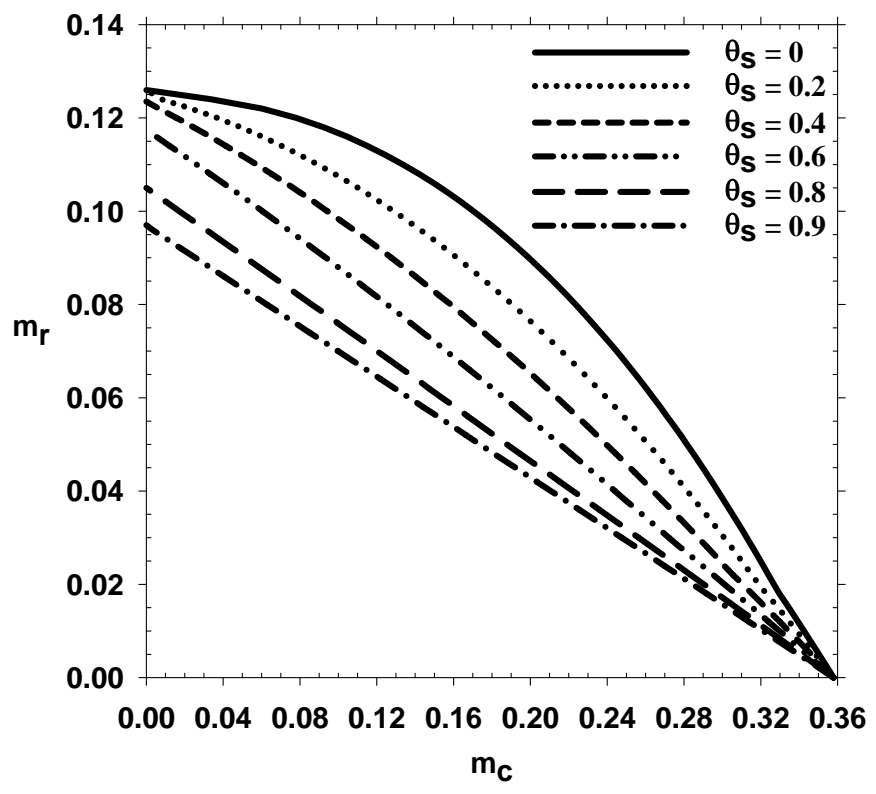

Fig. (12). The Optimmization Ranges of $m_{c}$ and $m_{r}$ as a Function of $\theta_{\mathrm{s}}$ for a Typical Trapezoidal Annular Fin of $\lambda=0.5\left(\beta=1, \mathrm{R}_{\mathrm{w}}=0.2\right.$, $\mathrm{V}=0.3$ ). new variable without changing the form of both differential equation and boundary conditions).

\subsubsection{Effects of Taper Ratio}

Besides the foregoing thermal parameters, the geometry parameters (volume, $\mathrm{V}$ and taper ratio, $\lambda$ ) also affect the optimization ranges. Even under the same thermal conditions, the availability of optimum designs will depend on the selection of geometry parameters.

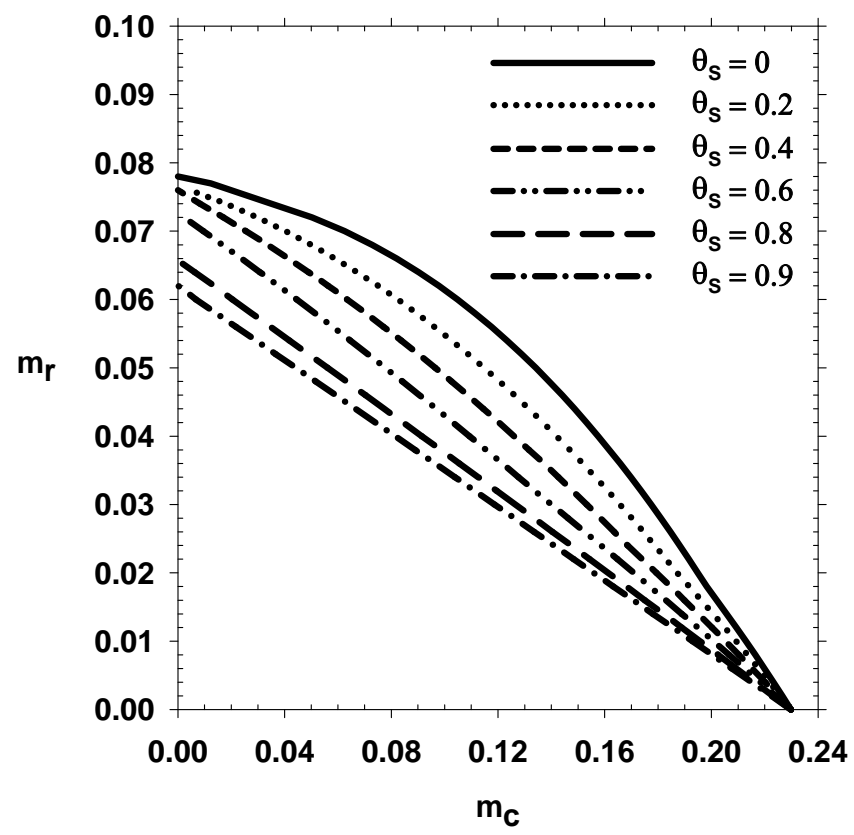

Fig. (13). The Optimization Ranges of $\mathrm{mc}$ and $\mathrm{mr}$ as a Function of $\theta_{\mathrm{s}}$ for a Rectangular Annular Fin $\left(\beta=1, \mathrm{R}_{\mathrm{w}}=0.2, \mathrm{~V}=0.3\right)$.

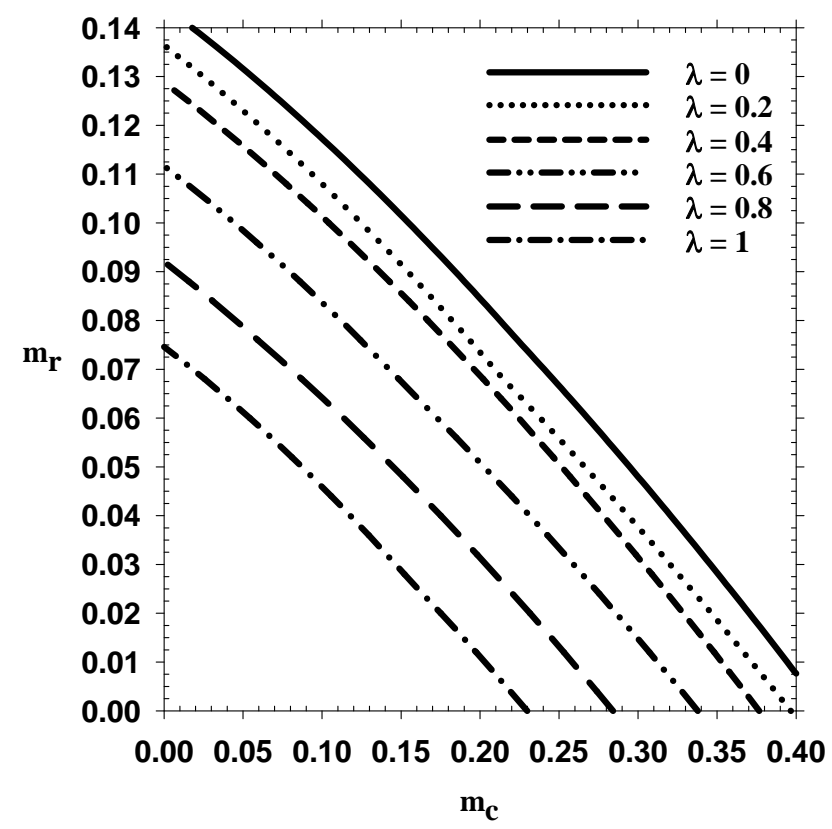

Fig. (14). The Optimization Ranges of $m_{c}$ and $m_{r}$ as a Function of $\lambda$ at $\mathrm{R}_{\mathrm{w}}=0.2\left(\beta=1, \theta_{\mathrm{s}}=0.5, \mathrm{~V}=0.3\right)$. 
The influences of the taper ratio $\lambda$ are shown in Figs. (14 and 15) with $R_{w}$ being 0.2 and 1.0 respectively. The optimization ranges for trapezoidal fins are bounded by those of rectangular and triangular fins. The triangular fin has the largest range while the rectangular fin has the smallest. In other words, for fins of fixed volume, the optimization ranges of $m_{c}$ and $m_{r}$ shrink with the increase of $\lambda$. This indicates that, for optimum trapezoidal fins, the

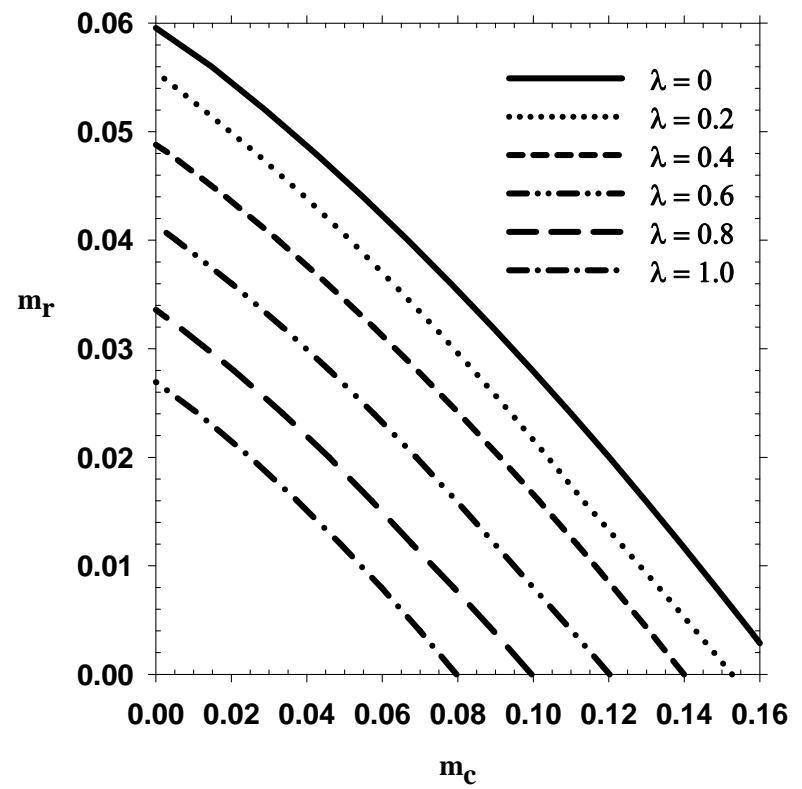

Fig. (15). The Optimization Ranges of $\mathrm{mc}$ and $\mathrm{mr}$ as a Function of $\lambda$ at $\mathrm{R}_{\mathrm{w}}=1.0$.

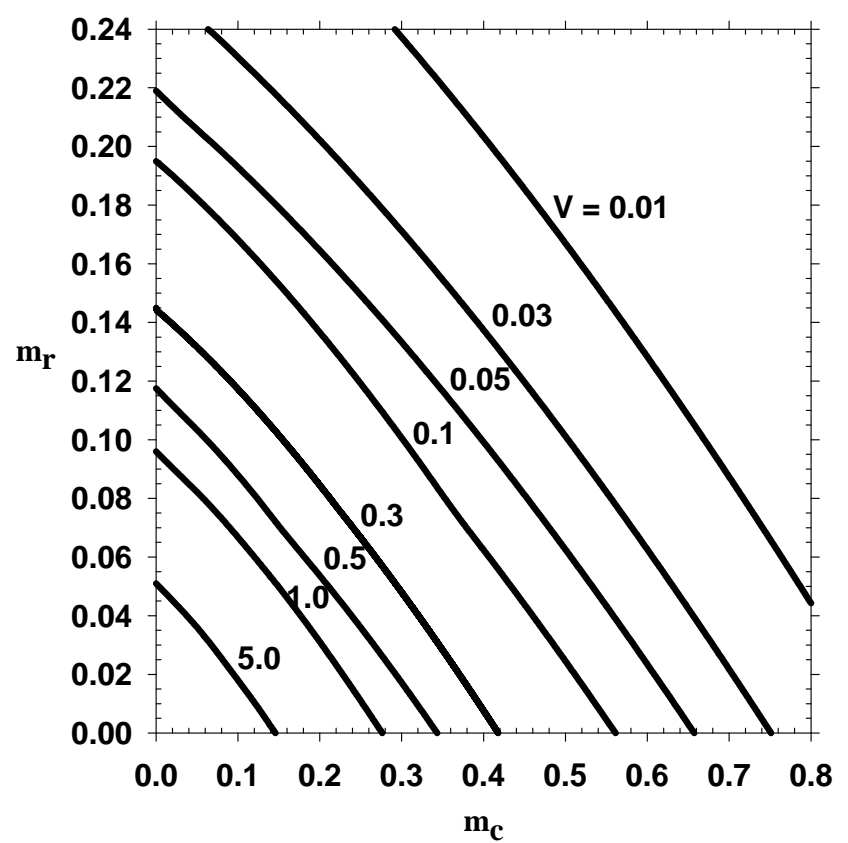

Fig. (16). The Optimization Ranges of $\mathrm{mm}_{\mathrm{c}}$ and $\mathrm{m}_{\mathrm{r}}$ as a Function of $\mathrm{V}$ for a Triangular Annular Fin $\left(\beta=1, \mathrm{R}_{\mathrm{w}}=0.2, \theta_{\mathrm{s}}=0.5\right)$. heat loss at the fin tip has a stronger influence on the optimization ranges than the length of arc effect, because, as mentioned earlier, triangular fins have no heat loss at the fin tip and rectangular fins do not have the length of arc effects.

\subsubsection{Effects of Fin Volume}

In Figs. (16-18), a wide range of fin volume (from 0.01 to 5 ) is chosen to evaluate how its variations will affect the

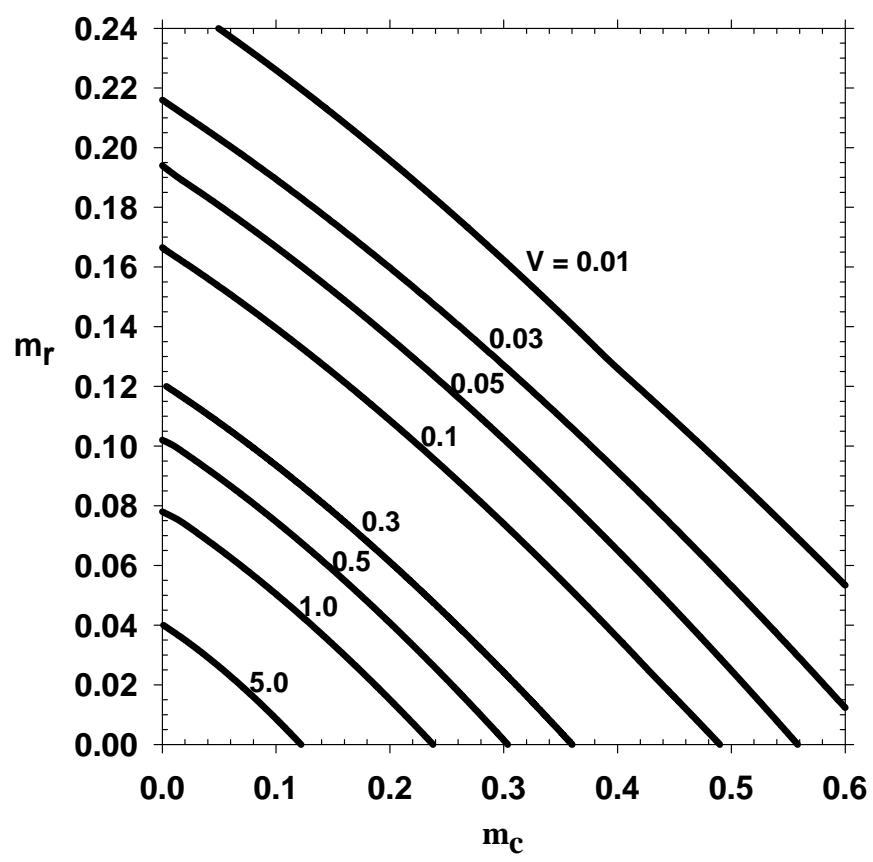

Fig. (17). The Optimization Ranges of $\mathrm{mc}$ and $\mathrm{mr}$ as a Function of $\mathrm{V}$ for a Typical Trapezoidal Annular Fin of $\lambda=0.5\left(\beta=1, \mathrm{R}_{\mathrm{w}}=0.2\right.$, $\left.\theta_{\mathrm{s}}=0.5\right)$.

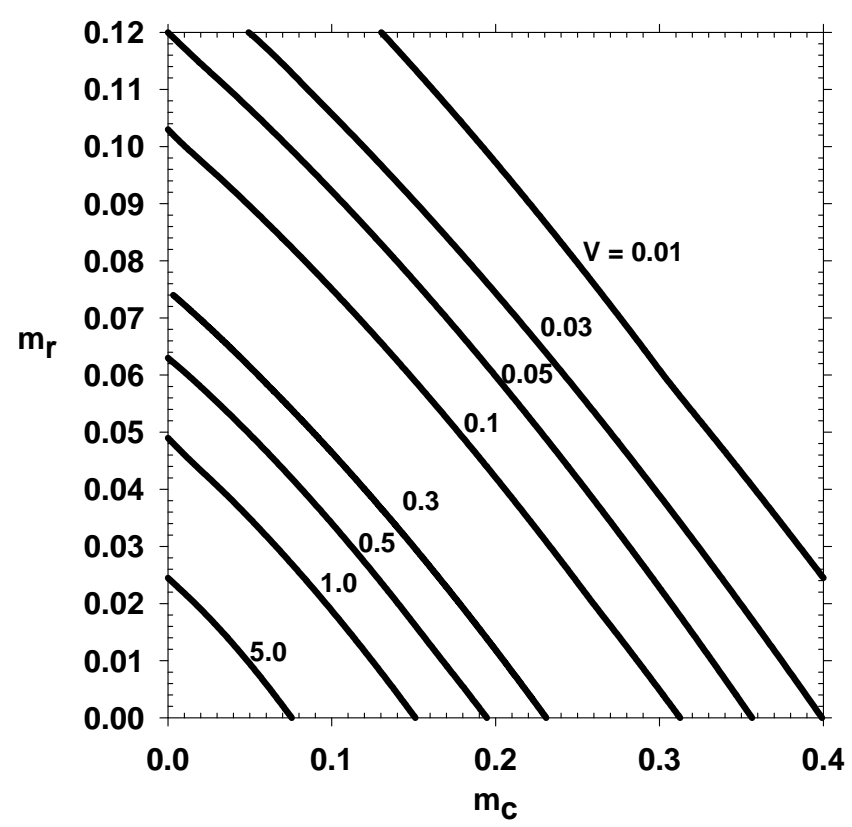

Fig. (18). The Optimizaation Ranges of $m_{c}$ and $m_{r}$ as a Function of $\mathrm{V}$ for a Rectangular Annular Fin $\left(\beta=1, \mathrm{R}_{\mathrm{w}}=0.2, \theta_{\mathrm{s}}=0.5\right)$. 
optimization ranges. It is observed that the optimization ranges of both $m_{c}$ and $m_{r}$ decrease with the increase of fin volume. The results suggest that the small fin volume will increase the availability of the optimum designs. Therefore, the optimum designs may not be obtainable if the volume is too big, especially when $m_{c}$ and $m_{r}$ are large.

\section{CONCLUSIONS}

The present numerical results reflect that the optimum fin design does not always exist. The length of arc effects, heat loss at the fin tip, overall wall resistance, Biot number and radiation number may create different optimization ranges or limitations. In this work, the ranges of fin optimum design under different thermal and geometrical conditions are investigated. Furthermore the present numerical solutions reveal that for a convective fin with negligible curvature, radiation and interfacial resistance effects, the optimum fin design always exists only when the fin tip is insulated. This finding creates a controversy in view of the well known Harper-Brown approximation [20] which has been widely cited in all undergraduate heat transfer textbooks. The approximation states that a convective fin tip can be always replaced by an insulated fin tip when the length of the fin is extended by one half of the fin thickness of the rectangular fin; this implies that a fin with a convective tip can always have optimum dimensions. While our solutions for the optimum fins indicate that the optimum design does not necessarily always exist if the fin tip is not insulated. We therefore conclude that the HarperBrown approximation should be used with caution. It is only valid for fin performance calculations but not for fin optimum design.

\section{CONFLICT OF INTEREST}

None declared.

\section{ACKNOWLEDGEMENT}

The assistance by Mr. Jay Liu on computer graphic is deeply appreciated.

\section{NOMENCLATURE}

$\begin{aligned} A & =\text { Fin geometry parameter } \\ m_{r} & =\text { Radiation characteristic number } \\ \mathrm{B} & =\text { Fin geometry parameter } \\ q & =\text { Heat transfer from the fin } \\ \mathrm{G} & =\text { Fin geometry parameter } \\ \mathrm{Q} & =\text { Dimensionless heat transfer from the fin } \\ h & =\text { Heat transfer coefficient over the fin surface } \\ r_{b} & =\text { Fin base radius } \\ h_{e} & =\text { Heat transfer coefficient at the fin tip }\end{aligned}$

$$
\begin{aligned}
r_{e} & =\text { Fin tip radius } \\
h_{f} & =\text { Heat transfer coefficient inside primary pipe } \\
R_{t c} & =\text { Contact thermal resistance } \\
k & =\text { Thermal conductivity of fin material } \\
R_{w} & =\text { Base wall thermal resistance } \\
k_{w} & =\text { Thermal conductivity of primary pipe } \\
T & =\text { Temperature along the fin } \\
m_{c} & =\text { Convection characteristic number over the fin } \\
\mathrm{v} & =\text { Furface } \\
\mathrm{V} & =\text { Dimensionless fin volume } \\
m_{c, e} & =\text { Convection characteristic number at the fin tip }
\end{aligned}
$$

\section{Greek Symbol}

$\beta=$ The ratio of convection characteristic numbers

$\rho=$ Fin radius ratio

$\sigma=$ Stefan-Boltzmann constant

$\varepsilon=$ Emissivity, dimensionless

$\xi=$ Dimensionless radius

$\delta_{b} \quad=\quad$ Fin base thickness

$\delta_{e} \quad=\quad$ Fin tip thickness

$\theta=$ Dimensionless temperature

$\lambda=$ Shape parameter, $\delta_{e} / \delta_{b}$

\section{Subscript}

$$
\begin{aligned}
\mathrm{a} & =\text { Ambient air } \\
b & =\text { Fin base } \\
e & =\text { Fin tip } \\
f & =\text { Fluid inside primary pipe } \\
i & =\text { Inside } \\
o & =\text { Outside } \\
s & =\text { Surroundings adjacent to fin }
\end{aligned}
$$

\section{Superscript}

$* \quad=$ Optimal value

\section{REFERENCES}

[1] E. Schmidt, Die Warmeubertragung Durch Rippen. Z. Ver. Deut. Ingenieure, vol.70, pp. 947-951, 1926.

[2] R.J. Duffin, “A variational problem relating to cooling of fins", $J$. Math. Mech. vol.8, pp. 47-56, 1959.

[3] C. J. Maday, "The minimum weight one-dimensional straight cooling fin”, ASME J. Engl. Ind., vol. 96, pp. 161-165, 1974. 
[4] L. Hanin, and A Campo, "A new minimum volume straight cooling fin taking into account the Length of Arc", Int. J. Heat Mass Transf, vol. 46, pp. 5145-5152, 2003.

[5] B. T. F. Chung, Z. Ma, and F. Liu, "General solutions for optimum dimensions of convective longitudinal fins with base wall thermal resistances", Proceedings of 11th Int. Heat Transfer Conference, Korea, vol. 5, pp. 357-362, 1998.

[6] P. Razelos, and K. Imre, "The optimum dimensions of circular fin with variable thermal parameters", J. Heat Transf., vol. 102, pp. 420-428, 1980.

[7] S. M. Zubair, and Jameel-ur-Rehman, Khan, "The optimal dimensions of convective-radiating circular fins", Heat Mass Transf., vol. 35, pp. 469-478, 1999

[8] A. Aziz, "Optimum dimensions of extended surfaces operating in a convective environment", Appl. Mech. Rev., vol. 45, pp. 155-173, 1992.

[9] B.T.F. Chung, and Y. Zhou, "Optimal design for convectingradiating annular fins of trapezoidal profile", Proceedings of 35th National Heat Transfer Conference, Anaheim, CA. Paper No. 12024, June 2001.

[10] A. Aziz, and A. D. Kraus, "Optimum design of radiating and convecting fins", Heat Transf. Eng., vol. 17, pp. 44-78, 1996.

[11] P Razelos, "A critical review of extended surface heat transfer", Heat Transf. Eng., vol.24, pp.11-28, 2003.
[12] A.D Kraus, A. Aziz, and J. Welty, Extended Surface Heat Transfer, John Wiley \& Sons Inc., New York, N.Y., 2001.

[13] K. Laor, and H., Kalman, "Performance and optimum dimensions of different cooling fins with a temperature-dependent heat transfer coefficient", Int. J. Heat Mass Transf., vol. 39, pp. 1993-2003, 1996.

[14] K. Laor, and H. Kalman, "The effect of tip Convection on the Performance and Optimum Dimensions of Cooling Fins", Int. Comm. Heat Mass Transf., vol. 19, pp. 569-584, 1992.

[15] B.T.F.Chung and J. Iyer, "Optimum dimensions of cooling fins", Heat Transf. Eng., vol. 14, pp. 31-42, 1993.

[16] R. H. Yeh, "Errors in one-dimensional fin optimization problem for convective heat transfer", Int. J. Heat Mass Transf., vol.39, pp. 3075-3078, 1996.

[17] A. Aziz, "Optimization of rectangular and triangular fins with convective boundary conditions", Int. Comm. Heat Mass Transf., vol. 12, pp. 479-482, 1985.

[18] R. W., Daniel, An Introduction to Numerical Methods and Optimization Techniques, Elsevier North-Holland Co: New York, 1978.

[19] D.Q. Kern and A.D. Kraus, Extended Surface Heat Transfer, McGraw-Hill Co: New York, 1972.

[20] B. Harper and D. R. Brown, Mathematical Equations for Heat Conduction in the Fins of Air-Cooled Engines, NACA Report 158, 1992 .

Received: June 21, 2011

Revised: September 25, 2011

Accepted: September 27, 2011

(c) Chung et al.; Licensee Bentham Open.

This is an open access article licensed under the terms of the Creative Commons Attribution Non-Commercial License (http://creativecommons.org/licenses/by-nc/3.0/) which permits unrestricted, non-commercial use, distribution and reproduction in any medium, provided the work is properly cited. 